\title{
Mechanism of endothelial nitric oxide synthase phosphorylation and activation by tentacle extract from the jellyfish Cyanea capillata
} Liming Zhang Corresp. 1,2

\footnotetext{
1 Marine Bio-pharmaceutical Institute, Second Military Medical University, Shanghai, China

2 Department of Marine Biotechnology,Faculty of Naval Medicine, Second Military Medical University, Shanghai, China

3 Department of Pharmacy,Changzheng Hospital, Second Military Medical University, Shanghai, China

Corresponding Authors: Xia Tao, Liming Zhang

Email address: taoxia2003@126.com, Imzhang@smmu.edu.cn
}

Our previous study demonstrated that tentacle extract (TE) from the jellyfish Cyanea capillata ( $C$. capillata) could cause a weak relaxation response mediated by nitric oxide (NO) using isolated aorta rings . However, the intracellular mechanisms of TE-induced vasodilation remain unclear. Thus, this study was conducted to examine the role of TE on Akt/eNOS/NO and $\mathrm{Ca}^{2+}$ signaling pathways in human umbilical vein endothelial cells (HUVECS) . Our results showed that TE induced dose- and time-dependent increases of eNOS activity and NO production. And TE also induced Akt and eNOS phosphorylation in HUVECs. However, treatment with specific PI3-kinase inhibitor (Wortmannin) significantly inhibited the increases in NO production and Akt/eNOS phosphorylation. In addition, TE also stimulated an increase in the intracellular $\mathrm{Ca}^{2+}$ concentration $\left(\left[\mathrm{Ca}^{2+}\right]_{\mathrm{i}}\right)$, which was significantly attenuated by either $\mathrm{IP}_{3}$ receptor blocker (Heparin) or PKC inhibitor (PKC 412). In contrast, extracellular $\mathrm{Ca}^{2+}$-free, L-type calcium channel blocker (Nifedipine), or PKA inhibitor (H89) had no influence on the $\left[\mathrm{Ca}^{2+}\right]_{\mathrm{i}}$ elevation. Since calcium ions also play a critical role in stimulating eNOS activity, we next explored the role of $\mathrm{Ca}^{2+}$ in TE-induced Akt/eNOS activation. In consistent with the results of $\left[\mathrm{Ca}^{2+}\right]_{i}$ elevation, we found that Akt/eNOS phosphorylation was also dramatically decreased by Heparin or PKC 412, but not affected by Nifedipine or H89. However, the phosphorylation level could also be decreased by the removal of extracellular calcium. Taken together, our findings indicated that TEinduced eNOS phosphorylation and activation were mainly through PI3K/Akt-dependent, $\mathrm{PKC} / \mathrm{IP}_{3} \mathrm{R}$-sensitive and $\mathrm{Ca}^{2+}$-dependent pathways. 
1 Mechanism of endothelial nitric oxide synthase phosphorylation and activation by tentacle

9

10

11

12

13

14

15 *Authors to whom correspondence should be addressed; E-mail address: taoxia2003@126.com

16 (X.Tao); lmzhang@smmu.edu.cn (L.Zhang.); Tel. /Fax: +86-21-8187-1128 (L.Zhang.).

${ }^{c}$ Department of Pharmacy, Changzheng Hospital, Second Military Medical University,

Shanghai, China
2

3 Beilei Wang ${ }^{\mathrm{a}, \mathrm{b}}$, Dan Liu ${ }^{\mathrm{b}}$, Chao Wang ${ }^{\mathrm{b}}$, Qianqian Wang ${ }^{\mathrm{a}, \mathrm{b}}$, Hui Zhang ${ }^{\mathrm{b}}$, Guoyan Liu ${ }^{\mathrm{a}, \mathrm{b}}$, Xia

4

5

6

7 b Department of Marine Biotechnology, Faculty of Naval Medicine, Second Military Medical

\section{extract from the jellyfish Cyanea capillata}

Tao $^{\mathrm{c}, *}$, Liming Zhang a, b,*

a Marine Bio-pharmaceutical Institute, Second Military Medical University, Shanghai, China
University, Shanghai, China 


\section{Abstract:}

19 Our previous study demonstrated that tentacle extract (TE) from the jellyfish Cyanea capillata (C. capillata) could cause a weak relaxation response mediated by nitric oxide (NO) using isolated aorta rings. However, the intracellular mechanisms of TE-induced vasodilation remain unclear. Thus, this study was conducted to examine the role of TE on Akt/eNOS/NO and

$23 \mathrm{Ca}^{2+}$ signaling pathways in human umbilical vein endothelial cells (HUVECs). Our results showed that TE induced dose- and time-dependent increases of eNOS activity and NO production. And TE also induced Akt and eNOS phosphorylation in HUVECs. However, treatment with specific PI3-kinase inhibitor (Wortmannin) significantly inhibited the increases in

27 NO production and Akt/eNOS phosphorylation. In addition, TE also stimulated an increase in the intracellular $\mathrm{Ca}^{2+}$ concentration $\left(\left[\mathrm{Ca}^{2+}\right]_{i}\right)$, which was significantly attenuated by either $\mathrm{IP}_{3}$ receptor blocker (Heparin) or PKC inhibitor (PKC 412). In contrast, extracellular $\mathrm{Ca}^{2+}$-free, Ltype calcium channel blocker (Nifedipine), or PKA inhibitor (H89) had no influence on the

$31\left[\mathrm{Ca}^{2+}\right]_{\mathrm{i}}$ elevation. Since calcium ions also play a critical role in stimulating eNOS activity, we

32 next explored the role of $\mathrm{Ca}^{2+}$ in TE-induced Akt/eNOS activation. In consistent with the results

33 of $\left[\mathrm{Ca}^{2+}\right]_{i}$ elevation, we found that Akt/eNOS phosphorylation was also dramatically decreased

34 by Heparin or PKC 412, but not affected by Nifedipine or H89. However, the phosphorylation

35 level could also be decreased by the removal of extracellular calcium. Taken together, our 
36 findings indicated that TE-induced eNOS phosphorylation and activation were mainly through

$37 \mathrm{PI} 3 \mathrm{~K} / \mathrm{Akt}$-dependent, $\mathrm{PKC} / \mathrm{IP}_{3} \mathrm{R}$-sensitive and $\mathrm{Ca}^{2+}$-dependent pathways. 


\section{Introduction}

Hypertension is the leading risk factor for cardiovascular diseases, causing almost $3.7 \%$ of

total disability-adjusted life-years and 13\% of all deaths (Park et al. 2015), which has also been a

serious public health burden all over the world. Thus, there is a critical need for developing anti-

43 hypertensive drugs to control the blood pressure. However, many synthetic antihypertensive

44 drugs are confirmed to have certain side effects, such as dizziness, headache, coughing,

45 angioedema, abnormal taste, and kidney and liver problems (Kuhlen \& Forcucci 2012).

46 Therefore, it is necessary to develop safer, more economical and innovative alternatives for the

47 prevention or treatment of hypertension.

48 Currently, many bioactive natural products, especially marine ones, have received

49 considerable attentions. Compared with terrestrial counterparts, marine organisms evolved a

50 stronger biological activity and a more complex structural diversity to adapt to the extreme

51 marine environment, such as high salt, high pressure, low-nutrient and unstable temperature

52 condition (Suleria et al. 2015). Therefore, marine natural products may become a novel

53 pharmaceutical resource to prevent and treat various diseases. In recent years, it was reported

54 that protein hydrolysates derived from several marine organisms, such as cod (Kim et al. 2000),

55 salmon (Ono et al. 2006), sea cucumber collagen (Zhao et al. 2009), shrimp (Zhang et al. 2009),

56 sesame (Biswas et al. 2010), squid skin (Lin et al. 2011), and jellyfish (Li et al. 2014; Liu et al. 
57 2012; Zhuang et al. 2012), could exert their hypotensive effects. Among them, jellyfish is one of

58 the most abundant resources in marine ecosystems and may provide many promising sources of

59 marine pharmaceuticals. In fact, the medicinal value of jellyfish has also been explored by

60 Chinese people for a long time. It is believed to be effective to patients with hypertension,

61 arthritis, ulcers and back pain. Besides, jellyfish can stimulate blood flow and reduce various

62 types of swellings (Liu et al. 2013; Liu et al. 2012). Furthermore, some jellyfish-derived proteins

63 have also been reported to possess antihypertensive (Li et al. 2014; Liu et al. 2012; Zhuang et al.

64 2012), antioxidant (Bruschetta et al. 2014), antimicrobial (Ayed et al. 2012) and anticoagulant

65 activities (Liu et al. 2015; Noguchi et al. 2005). Although jellyfish is traditionally recognized to

66 be beneficial in reducing blood pressure in China, its antihypertensive effect is rarely reported.

67 In our previous study, we demonstrated that tentacle extract (TE) from the jellyfish Cyanea

68 capillata (C. capillata) could cause a weak relaxation response in isolated aorta rings, which

69 might be mediated by nitric oxide (NO) (Wang et al. 2013a). However, the intracellular

mechanism of TE-induced vasodilation and its molecular cross-talk remain unclear. It is well

71 known that NO is produced by endothelial nitric oxide synthase (eNOS) in vascular endothelial

72 cells (Srivastava et al. 2012). eNOS is a calcium dependent enzyme and activated by the increase

73 in intracellular free calcium concentration $\left(\left[\mathrm{Ca}^{2+}\right]_{\mathrm{i}}\right)$ (Chen et al. 2010; Kerr et al. 2012), which is

74 induced either by an influx of extracellular calcium via channels such as voltage-dependent 
75

76

77 the activity of eNOS is also regulated by phosphorylation level. For example, eNOS

78

calcium channels, or by the release from intracellular stores in endoplasmic reticulum (ER) via receptors such as inositol 1,4,5-triphosphate receptors $\left(\mathrm{IP}_{3} \mathrm{Rs}\right)$ (Sammels et al. 2010). Besides, phosphorylation at Ser1177 by phosphatidylinositol 3-kinase (PI3-K)-dependent Akt plays a critical role in eNOS activation (Yoshitomi et al. 2011). Thus, the current study was designed to investigate the effects and molecular mechanisms of TE on eNOS activity in endothelial cells by detecting the changes in both intracellular $\mathrm{Ca}^{2+}$ concentration and Akt-dependent signal transduction pathways.

\section{Materials and methods}

\subsection{Drugs and chemicals reagents}

The HUVECs cell line was purchased from Zhongqiaoxinzhou Biotech (Shanghai, China).

MTT assay kit and NOS assay kit were purchased from Beyotime (Jiangsu, China). Human NO Elisa assay kit was purchased from Sangon Biotech (Shanghai, China). eNOS inhibitor $\mathrm{N}^{\omega}$-nitroL-arginine methyl ester (L-NAME) and PI3-K inhibitor Wortmannin were purchased from Sigma-Aldrich (Saint Louis, Missouri, America). The antibodies against phospho-Akt (Ser473), Akt, phospho-eNOS (Ser1177) and eNOS were purchased from Cell Signaling Technology

(Beverly, MA, America). The antibody against GAPDH was purchased from Abcam (Cambridge, MA, America). HRP-conjugated anti-rabbit IgG and anti-mouse IgG were purchased from 
93 Beyotime (Jiangsu, China). Fluo-4 AM was purchased from Invitrogen (California, America).

94 The stock solution of $1 \mathrm{mM}$ was prepared by adding dimethyl sulfoxide (DMSO) to solid powder.

95 The working solution of $5 \mu \mathrm{M}$ was prepared by adding serum free medium to the stock solution.

$961 \times$ HBSS (without phenol red, liquid, sterile-filtered) and $1 \times$ HBSS (without phenol red, 1.26

$97 \mathrm{mM} \mathrm{CaCl}_{2}$ ) were purchased from Sangon Biotech (Shanghai, China). L-type calcium channel

98 blocker Nifedipine, PKA inhibitor $\mathrm{H} 89, \mathrm{IP}_{3}$ receptor blocker Heparin and PKC inhibitor PKC

99412 were purchased from Sigma-Aldrich (Saint Louis, Missouri, America). In the measurement

100 of $\mathrm{Ca}^{2+}$ mobilization, Nifedipine solution of $100 \mu \mathrm{M}$ was prepared by $\mathrm{Ca}^{2+}$-containing HBSS,

101 whereas H89 solution of $10 \mu \mathrm{M}$, Heparin solution of $125 \mathrm{IU}$ and PKC 412 solution of $10 \mu \mathrm{M}$

102 were prepared by $\mathrm{Ca}^{2+}$-free HBSS.

103

104

105

106

107

108

109

110

\subsection{TE preparation from the jellyfish $C$. capillata}

Specimens of C. capillata were collected in June, 2014, in the Sanmen Bay, East China Sea, and identified by Professor Huixin Hong from the Fisheries College of Jimei University, Xiamen,

China. The removed tentacles were preserved in plastic bags on dry ice and immediately shipped

to Shanghai, where the samples were frozen at $-70{ }^{\circ} \mathrm{C}$ until use. TE was prepared following the method as described in previous reports (Bloom et al. 1998; Wang et al. 2013b). Briefly, frozen tentacles were thawed at $4{ }^{\circ} \mathrm{C}$ and immersed in filtered seawater at a mass/volume ratio of $1: 1$ to allow autolysis of the tissues for 4 days. The mixture was stirred for $30 \mathrm{~min}$ twice daily. The 
111 autolyzed mixture was centrifuged at $10,000 \times g$ for $15 \mathrm{~min}$, thrice. The resultant supernatant

112 was the TE. All procedures were performed at $4{ }^{\circ} \mathrm{C}$ or in an ice bath. The TE was centrifuged at

$11310,000 \times g$ for $15 \mathrm{~min}$ to remove the sediments, followed by dialysis against phosphate buffered

114 saline (PBS, $0.01 \mathrm{~mol} / \mathrm{L}, \mathrm{pH} \mathrm{7.4)}$ for over $8 \mathrm{~h}$ before use. The protein concentration in the

115 preparations was determined using the method of Bradford.

\section{2.3. Endothelial cell cultures}

(Hyclone, Waltham, MA, America) supplemented with 10\% fetal bovine serum (FBS, Gibco,

California, America), $100 \mathrm{U} / \mathrm{ml}$ penicillin and $100 \mu \mathrm{g} / \mathrm{ml}$ streptomycin at $37^{\circ} \mathrm{C}$ in a humidified

incubator with $95 \%$ air and $5 \% \mathrm{CO}_{2}$.

\subsection{Cell viability assay}



according to manufacturer's instructions (NOS assay kit). Briefly, cells were exposed to $100 \mu \mathrm{l}$ reaction buffer solutions and subsequent $100 \mu \mathrm{l}$ reaction solutions for $2 \mathrm{~h}$ with/without eNOS inhibitor (L-NAME). Then the plates were observed on a microplate reader (BioTek, Winooski, VT, USA) at excitation/emission wavelengths of 495/515 $\mathrm{nm}$. The activity of eNOS was calculated by the following equation: Relative activity (eNOS) $=\left(\mathrm{RFU}_{\text {stimulated }}\right.$ $\left.\mathrm{RFU}_{\text {inhibitor+stimulated }}\right) /\left(\mathrm{RFU}_{\text {unstimulated }}-\mathrm{RFU}_{\text {inhibitor+unstimulated }}\right)$.

\subsection{Measurement of NO concentration in HUVECs} incubated with different concentrations of TE $(0-4 \mu \mathrm{g} / \mathrm{ml})$ at $37^{\circ} \mathrm{C}$ for $1 \mathrm{~h}$. In the time-effect experiments, cells were incubated with TE $(1 \mu \mathrm{g} / \mathrm{ml})$ for different time durations $(0-180 \mathrm{~min})$ at

144 Wortmannin for $15 \mathrm{~min}$ before treatment with $\mathrm{TE}(1 \mu \mathrm{g} / \mathrm{ml}, 1 \mathrm{~h})$. After incubation, culture supernatants were collected, and NO concentration was measured using a microplate reader

146 (BioTek, Winooski, VT, America) in the absorbance value at $450 \mathrm{~nm}$ according to 
147 manufacturer's instructions (Human NO Elisa assay kit).

\section{2.7. Western blotting}

149 In the time-effect group, HUVECs were treated with TE $(1 \mu \mathrm{g} / \mathrm{ml})$ for different time

150 durations (0-60 min). In PI3K/Akt-dependent signaling pathways, HUVECs were treated with

$151 \mathrm{TE}(1 \mu \mathrm{g} / \mathrm{ml})$ for $30 \mathrm{~min}$ in the presence or absence of Wortmannin. In calcium-dependent

152 signaling pathways, HUVECs were treated with TE $(1 \mu \mathrm{g} / \mathrm{ml})$ for $30 \mathrm{~min}$ in the presence of

153 extracellular $\mathrm{Ca}^{2+}$-containing, Nifedipine, extracellular $\mathrm{Ca}^{2+}$-free, $\mathrm{H} 89$, Heparin and PKC 412,

154 respectively.

After treatments, cells were lysed on ice in RIPA buffer with protease inhibitor (1\% PMSF).

The protein content of the lysate was measured by the method of Bradford. Equal amounts of

157 protein per sample were loaded on 10\% SDS-PAGE gels and then transferred to the

158 nitrocellulose membranes. The membranes were subsequently blocked with 5\% non-fat dry milk

159 in TBST (3 g Tris-base, $8 \mathrm{~g} \mathrm{NaCl}, 0.2 \mathrm{~g} \mathrm{KCl}, 0.05 \%$ Tween-20, dilute with water to $1000 \mathrm{ml}, \mathrm{pH}$

7.4) for $2 \mathrm{~h}$ at room temperature. Then, the samples were incubated overnight at $4^{\circ} \mathrm{C}$ with

primary antibodies as follows: p-Akt (1:1000), Akt (1:1000), p-eNOS (1:500), eNOS (1:500) and

162 GAPDH (1: 5000), with gentle shaking. The ECL method was used with secondary antibodies

163 (HRP-conjugated anti-rabbit IgG and anti-mouse $\mathrm{IgG}$ ) at a dilution of 1:5,000 for $2 \mathrm{~h}$ at room 
165 (Syngene G: Box, America). Quantitative densitometric analyses of immunoblots were 166 performed using an ImageJ software (Ver. 1.48), and the relative ratio was calculated.

167

168

169

170

171

172

173

174

175

176

177

178

179

180

181

182

\subsection{Measurement of $\mathrm{Ca}^{2+}$ mobilization}

HUVECs were cultured in a confocal dish (Coverglass Bottom Dish, Corning, New York, America) and serum-starved overnight before use. Cells were loaded with Fluo-4 AM working solution $(5 \mu \mathrm{M})$ at $37{ }^{\circ} \mathrm{C}$ in the dark for $30 \mathrm{~min}$, then washed three times with $\mathrm{Ca}^{2+}$-free HBSS to remove excess extracellular dye.

To characterize the possible contribution of extracellular calcium, the cells were pre-treated with $1.26 \mathrm{mM} \mathrm{Ca}^{2+}$-containing HBSS with/without Nifedipine $(100 \mu \mathrm{M})$ for $15 \mathrm{~min}$ before the addition of $\mathrm{TE}(10 \mu \mathrm{g} / \mathrm{ml}$ final concentration). To examine the possible participation of intracellular calcium stores, the cells were pre-treated with $\mathrm{Ca}^{2+}$-free $\mathrm{HBSS}$ in the presence or absence of H89 $(10 \mu \mathrm{M})$, Heparin (125 IU) or PKC $412(10 \mu \mathrm{M})$ for 15 min before the addition of TE $(10 \mu \mathrm{g} / \mathrm{ml}$ final concentration). All the concentrations of the inhibitors were screened out and proved effective in similar preparations of the preliminary experiments. confocal microscope (Olympus FV 1000, Japan) with excitation and emission wavelengths of $488 \mathrm{~nm}$ and $526 \mathrm{~nm}$, respectively. Fluorescence images were recorded as time-series mode per $10 \mathrm{~s}$ intervals (100 images altogether). TE was added after the first five images were collected. 
183 Fluorescence intensities were obtained from the data set of images using FV10-ASW 3.1

184 (Olympus, Japan).

185 2.9. Statistical analysis

186 In the experiments, the images shown were the representatives of at least three experiments

187 performed on different experimental days. Data were presented as mean \pm SEM. Analysis of 188 variance (ANOVA) and Student's t-test were used in statistical evaluation of the data as

189 appropriate. $P$-values less than or equal to 0.05 were considered significant.

190

\section{Results}

\subsection{Effects of TE on the viability of HUVECs}

192

As shown in Fig. 1, when HUVECs were treated with various doses of TE for $1 \mathrm{~h}$, the

viability was decreased at the concentrations of $12-24 \mu \mathrm{g} / \mathrm{ml}$ in a dose-dependent manner, and

the $\mathrm{IC}_{50}$ was $15.55 \mu \mathrm{g} / \mathrm{ml}$. However, $\mathrm{TE}$ at $0.5-8 \mu \mathrm{g} / \mathrm{ml}$, did not significantly influence the cell

viability after $1 \mathrm{~h}$ treatment. Similarly, after treatment with TE for $6 \mathrm{~h}$, the viability of cells was

the relatively safe dose of TE less than $8 \mu \mathrm{g} / \mathrm{ml}$ was selected for the next study. 
199

200

201

202

203

204

205

206

207

208

209

210

211

212

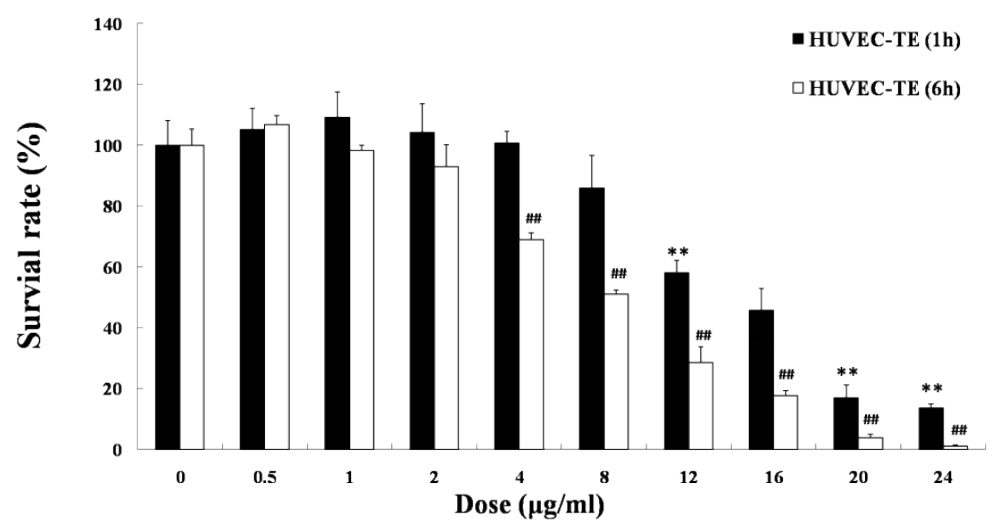

Fig. 1 Effects of TE on the viability of HUVECs. HUVECs were treated with the following agents: $0-24 \mu \mathrm{g} / \mathrm{ml}$ of TE. The MTT assays were performed at $1 \mathrm{~h}$ and $6 \mathrm{~h}$ after treatments. Each column represents mean $\pm \mathrm{SD}$ of six samples. ${ }^{* *} P<0.01$ vs. Control $(1 \mathrm{~h}),{ }^{\#} P<0.01$ vs. Control $(6 \mathrm{~h})$.

\subsection{Effect of TE on the eNOS activity and NO production in HUVECs}

Treatments with various doses of TE $(0-4 \mu \mathrm{g} / \mathrm{ml})$ increased the activity of eNOS in a concentration-dependent manner within $1 \mathrm{~h}$ in HUVECs: a significant increase was induced at the concentration of $0.5 \mu \mathrm{g} / \mathrm{ml}$ and the peak at $4 \mu \mathrm{g} / \mathrm{ml}$ (Fig. 2A). Similarly, TE-induced NO production was also concentration-dependent within $1 \mathrm{~h}$ : a significant increase was induced at the concentration of $1 \mu \mathrm{g} / \mathrm{ml}$ and the peak at $4 \mu \mathrm{g} / \mathrm{ml}$ (Fig. 2C). So the dose of $1 \mu \mathrm{g} / \mathrm{ml}$ was used in the next time-effect experiments. It was also showed a significant time-dependent increase was induced by TE $(1 \mu \mathrm{g} / \mathrm{ml})$ in the activity of eNOS (Fig. 2B) and NO production (Fig. 2D), which reached the maximum at about $1 \mathrm{~h}$ and maintained until $3 \mathrm{~h}$. Therefore, the concentration 
213 of $1 \mu \mathrm{g} / \mathrm{ml}$ and the time of $1 \mathrm{~h}$ were selected for the next study on PI3K/Akt/eNOS signaling

214 pathways.
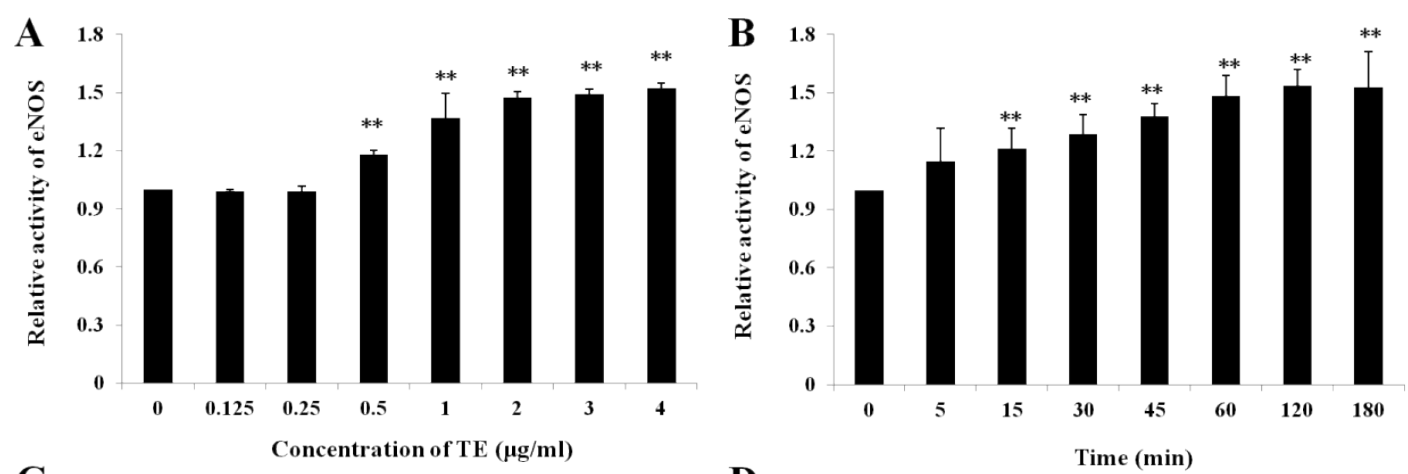

C ${ }_{120}$

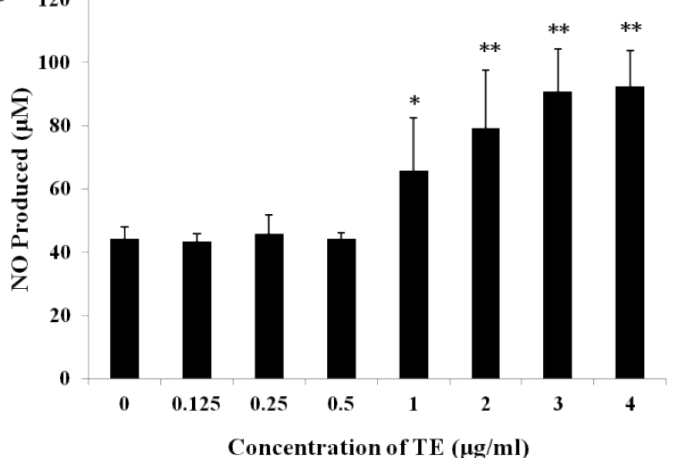

D

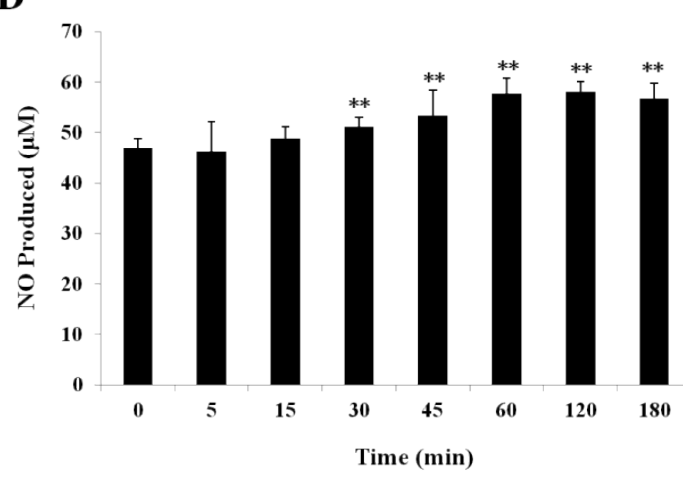

215

216 Fig. 2 Effects of TE on relative activity of eNOS and NO production in HUVECs. A and C:

217 HUVECs were treated with various dose of TE $(0-4 \mu \mathrm{g} / \mathrm{ml})$ for $1 \mathrm{~h}$. B and D: HUVECs were

218 treated with TE $(1 \mu \mathrm{g} / \mathrm{ml})$ for various time $(0-180 \mathrm{~min})$. Each column represents mean $\pm \mathrm{SD}$ of

219 six samples. ${ }^{* *} P<0.01,{ }^{*} P<0.05$ vs. Control.

\subsection{Effect of TE on the PI3K/Akt/eNOS signaling pathway in HUVECs}

We next examined the phosphorylation of Akt and eNOS after the stimulation with TE (1

$222 \mu \mathrm{g} / \mathrm{ml}$ ). As shown in Fig. 3A, Akt phosphorylation was significantly induced by TE from 15 to 
224 no effect on the level of total Akt. Similarly, eNOS phosphorylation was also induced by TE

225 from 15 to $60 \mathrm{~min}$, with the maximum phosphorylation at about $30 \mathrm{~min}$, while TE had no effect

226 on the level of total eNOS, either. These results indicated that TE could activate the Akt/eNOS

227 pathways. On the other hand, since Akt had been reported to phosphorylate eNOS via PI3-kinase,

228 we then pretreated HUVECs with the PI3-K inhibitor Wortmannin $(10 \mu \mathrm{M})$ before TE to

229 investigate whether this upstream signaling pathway was involved. As shown in Fig. 3B, the

230 inhibition of PI3-kinase completely blocked the TE-induced Akt/eNOS phosphorylation, thus

231 demonstrating the requirement for this kinase during the Akt/eNOS activation by TE.

$\mathbf{A}$
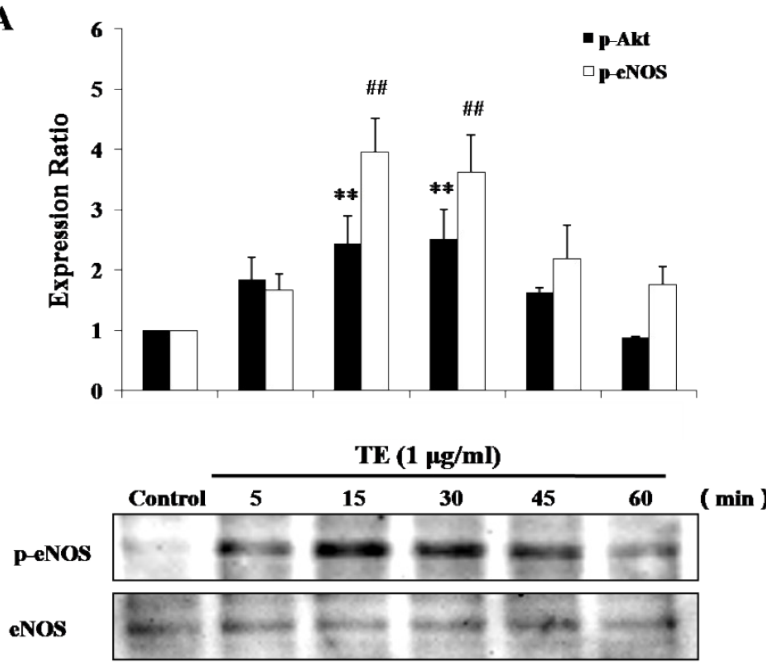

p-Akt
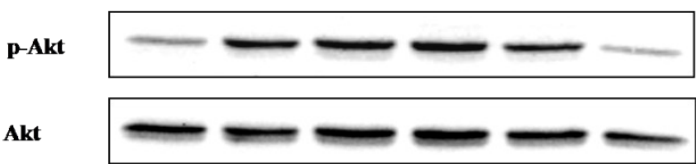

GAPDH

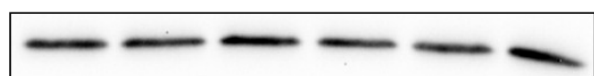

B
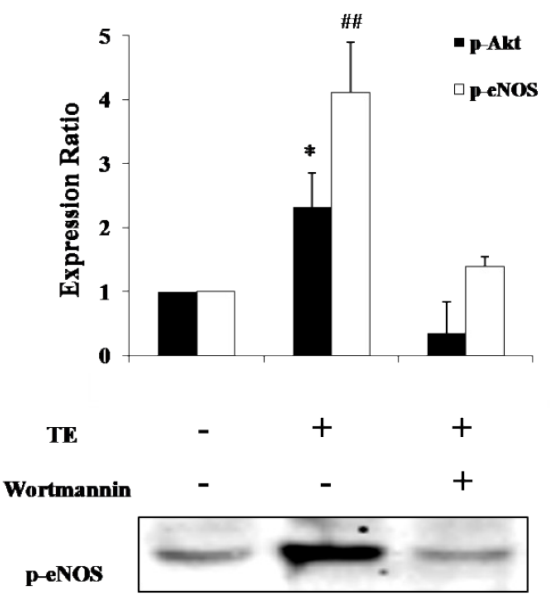

232

Fig. 3 Responses of the PI3K/Akt/eNOS signaling pathway to TE $(1 \mu \mathrm{g} / \mathrm{ml})$. A: HUVECs were

234 treated with TE $(1 \mu \mathrm{g} / \mathrm{ml})$ for various time durations. B: HUVECs were treated with TE $(1 \mu \mathrm{g} / \mathrm{ml})$ 
235 in the presence or absence of Wortmannin (PI3-K inhibitor) for $30 \mathrm{~min} .{ }^{*} P<0.05,{ }^{* *} P<0.01$ vs.

236 p-Akt/Akt of Control. ${ }^{\#} P<0.01$ vs. p-eNOS/eNOS of Control.

237

238

239

240

241

242

243

244

245

246

247

248

\subsection{Effect of PI3K/Akt/eNOS pathway inhibition on the TE-induced NO production}

As shown in Fig. 4, both eNOS inhibitor L-NAME and PI3-K inhibitor Wortmannin resulted in a significant reduction in TE-induced NO production, which indicated that PI3K/Akt/eNOS mediated the release of NO induced by TE, suggesting an important role of the PI3K/Akt/eNOS pathway in TE-induced NO release.

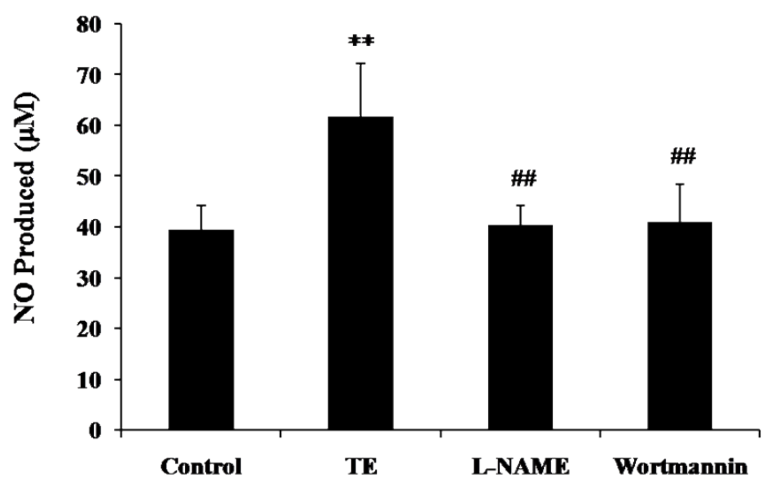

Fig. 4 Effects of PI3K/Akt/eNOS inhibitors on TE-induced NO production. HUVECs were treated with TE $(1 \mu \mathrm{g} / \mathrm{ml})$ in the presence or absence of eNOS inhibitor (L-NAME) or PI3-K inhibitor (Wortmannin) for $1 \mathrm{~h} .{ }^{* *} P<0.01$ vs. Control, ${ }^{\# \#} P<0.01$ vs. TE.

\subsection{Effects of TE on intracellular $\mathrm{Ca}^{2+}$ concentration changes in HUVECs}

To explore the effects of TE upon the $\mathrm{Ca}^{2+}$ concentration in HUVECs, we incubated the cells with the $\mathrm{Ca}^{2+}$ indicator dye Fluo-4 AM, and then stimulated them with TE whilst the time- 
249 series images of intracellular $\mathrm{Ca}^{2+}$ levels were detected. As shown in Fig. 5, TE could induce a 250 rapid rise in the intracellular $\mathrm{Ca}^{2+}$ concentration $\left(\left[\mathrm{Ca}^{2+}\right]_{\mathrm{i}}\right)$, which peaked within 1 min and 251 sustained for about $10 \mathrm{~min}$. Secondly, to determine the involvement of extracellular calcium, we 252 next used the L-type calcium blocker Nifedipine (in $\mathrm{Ca}^{2+}$-containing HBSS) and $\mathrm{Ca}^{2+}$-free HBSS 253 instead of the normal HBSS for the examination of TE-induced $\left[\mathrm{Ca}^{2+}\right]_{i}$ changes. Our results 254 showed that neither Nifedipine nor extracellular $\mathrm{Ca}^{2+}$-free had influences on the TE-induced $255\left[\mathrm{Ca}^{2+}\right]_{\mathrm{i}}$ rise, which indicated that TE-induced $\left[\mathrm{Ca}^{2+}\right]_{\mathrm{i}}$ rise did not come from the extracellular $\mathrm{Ca}^{2+}$ 256 influx, but mainly from the intracellular stored $\mathrm{Ca}^{2+}$ release. So, to further explore the source of $257 \mathrm{Ca}^{2+}$, we next investigated the effects of PKA inhibitor $\mathrm{H} 89, \mathrm{IP}_{3}$ receptor blocker Heparin and 258 PKC inhibitor PKC 412 on the $\left[\mathrm{Ca}^{2+}\right]_{\mathrm{i}}$ elevation. Our results showed that the TE-induced $\left[\mathrm{Ca}^{2+}\right]_{\mathrm{i}}$ 259 rise was significantly attenuated either by Heparin or by PKC 412, but not affected by H89, 260 suggesting that the $\mathrm{IP}_{3} \mathrm{R}$ and $\mathrm{PKC}$ signaling play major roles in the TE-induced $\left[\mathrm{Ca}^{2+}\right]_{\mathrm{i}}$ elevation. 

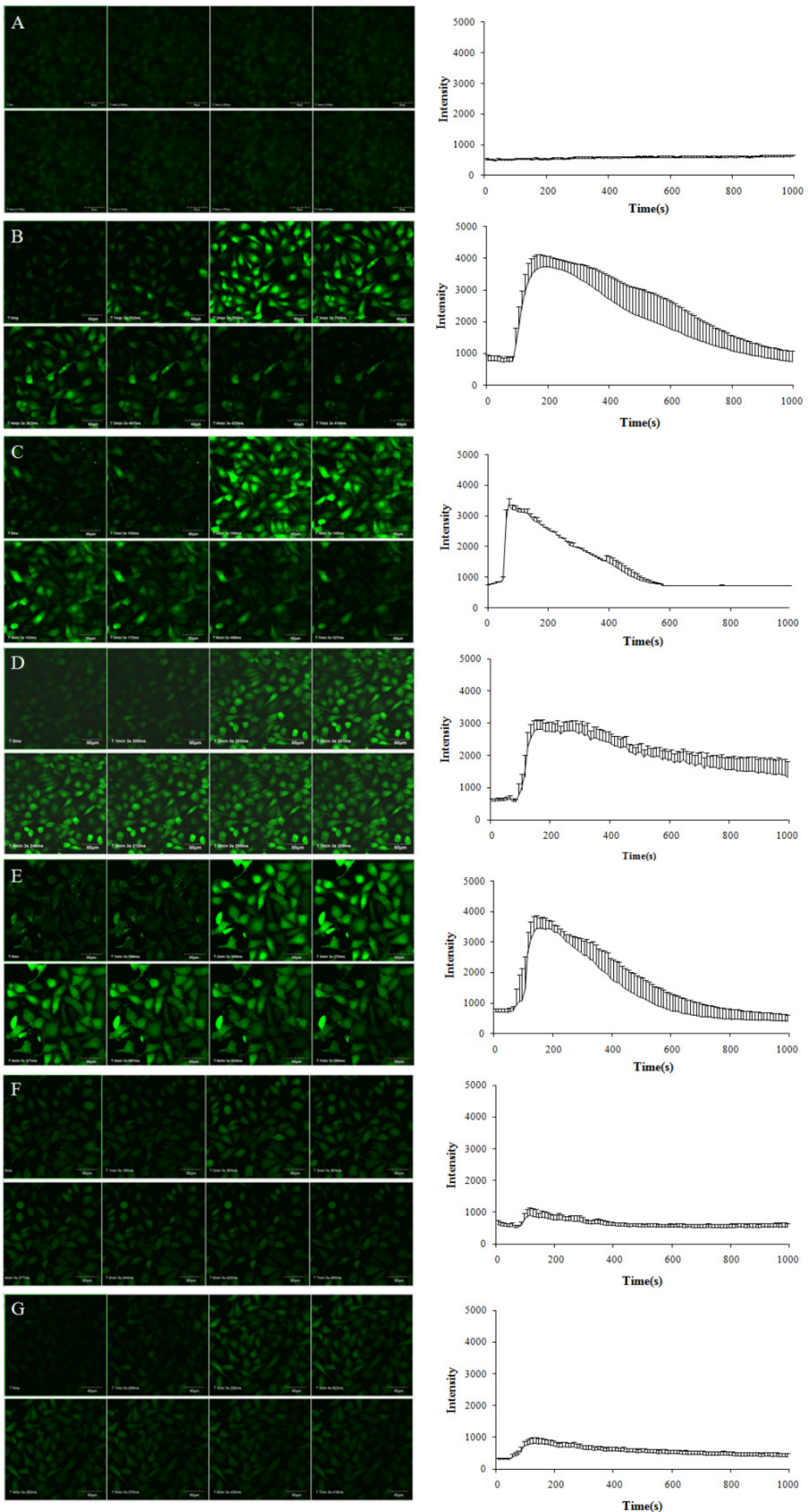

262 Fig. 5 Characterization of the $\mathrm{Ca}^{2+}$ concentration evoked by $\mathrm{TE}(10 \mu \mathrm{g} / \mathrm{ml})$ in Fluo-4-loaded

263 HUVECs. Left panel: Experimental records of the $\mathrm{Ca}^{2+}$ fluorescence image. Right panel: 
264 Statistical results of the $\mathrm{F}_{488} / \mathrm{F}_{526}$ ratio. A: Control; B: incubated with $\mathrm{Ca}^{2+}$-containing $\mathrm{HBSS}$; C:

265 incubated with $\mathrm{Ca}^{2+}$-containing HBSS plus Nifedipine; D: incubated with $\mathrm{Ca}^{2+}$-free HBSS; E:

266 incubated with $\mathrm{Ca}^{2+}$-free HBSS plus H89; F: incubated with $\mathrm{Ca}^{2+}$-free HBSS plus Heparin; G:

267 incubated with $\mathrm{Ca}^{2+}$-free HBSS plus $\mathrm{PKC} 412$.

\section{3.6. Effect of calcium signaling on the TE-induced Akt/eNOS activation}

the role of $\mathrm{Ca}^{2+}$ in the activation of Akt/eNOS pathway in response to TE. As shown in Fig. 6,

we indeed found the evidence for a critical role of $\mathrm{Ca}^{2+}$ in the TE-induced Akt/eNOS activation.

Firstly, we found that TE (in $\mathrm{Ca}^{2+}$-containing HBSS) could induce the phosphorylation of both

Akt and eNOS, while the phosphorylation level was dramatically decreased by the removal of

extracellular calcium, indicating that $\mathrm{Ca}^{2+}$ was essential to the TE-induced Akt/eNOS activation.

Secondly, to further elucidate the contribution of extracellular and intracellular calcium to the

effects of TE-induced Akt/eNOS activation, we similarly performed the Akt/eNOS activity

H89 $(10 \mu \mathrm{M}), \mathrm{IP}_{3}$ receptor blockers Heparin (125 IU) and PKC inhibitor PKC $412(10 \mu \mathrm{M})$,

respectively. As shown in Fig. 6, the phosphorylation of Akt/eNOS was completely attenuated

by the incubation with Heparin or PKC 412, which indicated that the Akt/eNOS activation might 
282

283 284 285 $\mathrm{Ca}^{2+}$ signaling.

$\mathbf{A}$
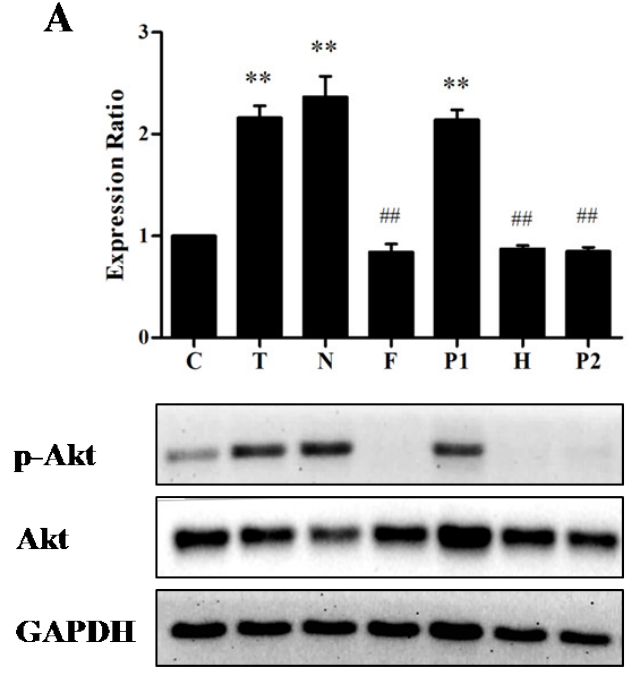

B
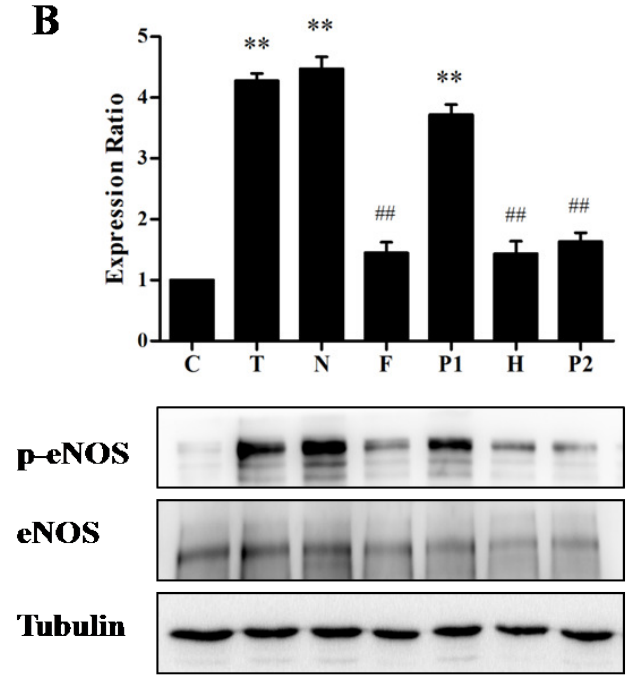

286

287 Fig. 6 Effects of $\mathrm{Ca}^{2+}$ signaling on TE-induced Akt/eNOS activation. HUVECs were pretreated for 15 min with HBSS ( $\mathrm{Ca}^{2+}$-containing), Nifedipine (in $\mathrm{Ca}^{2+}$-containing $\mathrm{HBSS}$ ), $\mathrm{HBSS}$ (in $\mathrm{Ca}^{2+}$ free HBSS), $\mathrm{H} 89$ (in $\mathrm{Ca}^{2+}$-free HBSS), Heparin (in $\mathrm{Ca}^{2+}$-free $\mathrm{HBSS}$ ), PKC 412 (in $\mathrm{Ca}^{2+}$-free

291 (Ser473) were observed by immunoblotting with a phospho-specific antibody. B: eNOS and phospho-eNOS (Ser1177) were assayed by immunoblotting with a phospho-specific antibody. ${ }^{* *} P<0.01$ vs. Control. ${ }^{\# \#} P<0.01$ vs. TE $\left(\mathrm{Ca}^{2+}\right) . \mathrm{C}:$ Control, T: TE $\left(\mathrm{Ca}^{2+}\right), \mathrm{N}:$ Nifedipine, F: TE ( $\mathrm{Ca}^{2+}$-free), P1: PKA inhibitor (H89), H: Heparin, P2: PKC inhibitor (PKC 412). 
295

296

297

298

299

300

301

302

303

304

305

306

307

308

309

310

311

312

\section{Discussion}

cardiovascular characterization of jellyfish venoms. However, the current studies have been

hindered by some reasons: (1) jellyfish venoms are sticky, thermolabile and difficult to be separated (Feng et al. 2010); and (2) venom samples are difficult to be collected due to the small

amount of the venoms in nematocysts (Xiao et al. 2009). To solve the problem, we have

previously compared the nematocyst venoms with TE (devoid of nematocysts) from the jellyfish

C. capillata. Our result suggested that TE may serve as a potential alternative of the nematocyst

venoms with much richer source for isolating and purifying cardiovascular active proteins (Xiao

et al. 2009), because these proteins in the nematocyst venoms and TE are probably encoded by

the same gene fragment (Nagai et al. 2000a; Nagai et al. 2000b). Besides, using isolated rat

aortic rings, we also verified that TE did have a direct vascular activity. Our results showed that

TE could cause a weak relaxation response, which was significantly attenuated either by the

removal of the endothelium, or by the blockade of NO synthase by L-NAME (Wang et al.

2013a), suggesting that the vasodilation induced by TE was possibly mediated by an NO-

dependent pathway. So in the current study, we subsequently measured the eNOS activity and

NO concentration induced by TE in HUVECs. Our results showed that TE could induce concentration- and time-dependent increases in eNOS activity and NO production in HUVECs; 
313 in addition, eNOS inhibitor L-NAME completely attenuated NO production induced by TE,

314 confirming that TE-induced vasodilative effects were mainly mediated by the release of NO via

315 the activation of eNOS in the endothelial cells.

316 It is well-established that eNOS is tightly regulated not only at the transcriptional level but

317 also by certain post-transcriptional mechanisms (Vilahur et al. 2014; Yoshitomi et al. 2011). In

318 the present study, we found that TE did not have an effect on the level of eNOS protein, but did

319 markedly induce eNOS phosphorylation at Ser1177 from 15 to $60 \mathrm{~min}$, suggesting that TE

320 induced an increase in eNOS activity at the post-transcriptional level in HUVECs. To further

321 explore the possible mechanisms underlying eNOS activation in HUVECs after treatment with

322 TE, we next investigated the potential role of PI3K/Akt-dependent signaling. Our results

323 demonstrated that the PI3K/Akt pathway is necessary for eNOS activation in TE-treated

324 HUVECs. Several lines of evidence supported this notion: (1) TE stimulated Akt

325 phosphorylation from 15 to $45 \mathrm{~min}$, which occurred slightly preceding eNOS phosphorylation; (2)

326 PI3-kinase inhibitor Wortmannin, not only blocked TE-evoked Akt, but also inhibited TE-

327 induced Ser1177 phosphorylation of eNOS under the same condition; (3) Wortmannin

328 completely attenuated TE-induced NO production, consistent with the effects of the eNOS

329 inhibitor L-NAME.

On the other hand, since calcium ions play a crucial role in stimulating eNOS activity 
331 through a $\mathrm{Ca}^{2+} /$ calmodulin-dependent mechanism (Chen et al. 2010), we next explored the role

332 of $\mathrm{Ca}^{2+}$ in the activation of eNOS in response to TE. Firstly, we assessed the effects of TE on

333 intracellular $\left[\mathrm{Ca}^{2+}\right]_{\mathrm{i}}$ in HUVECs using the calcium specific fluorescent dye, Fluo-4 AM, and

334 found that TE did induce a rapid rise in the intracellular $\left[\mathrm{Ca}^{2+}\right]_{\mathrm{i}}$, which peaked within 1 min and

335 sustained for about $10 \mathrm{~min}$. Secondly, since $\mathrm{Ca}^{2+}$ is maintained by two mechanisms: entry from

336 extracellular medium through the opening of calcium permeable channels in plasma membrane,

337 or release from intracellular organelles (mainly ER), we further explored the source of TE-

338 evoked $\mathrm{Ca}^{2+}$ rise using $\mathrm{Ca}^{2+}$ signaling related inhibitors. Our results showed that neither

339 Nifedipine nor extracellular $\mathrm{Ca}^{2+}$-free had a significant influence on the TE-induced $\left[\mathrm{Ca}^{2+}\right]_{\mathrm{i}}$ rise,

340 indicating that TE-induced $\left[\mathrm{Ca}^{2+}\right]_{\mathrm{i}}$ rise did not come from the extracellular $\mathrm{Ca}^{2+}$ influx, but

341 mainly from the intracellular stored $\mathrm{Ca}^{2+}$ release. In $\mathrm{ER}$, the $\mathrm{IP}_{3} \mathrm{R}$ channel is capable of releasing

342 a large quantity of $\mathrm{Ca}^{2+}$ to the cytosol, and is believed to play a primary role in $\mathrm{Ca}^{2+}$ mobilization

343 (Morgado et al. 2012; Tiruppathi et al. 2002). This study also showed that $\mathrm{Ca}^{2+}$ elevation was

344 significantly attenuated by the blockade of $\mathrm{IP}_{3} \mathrm{R}$ by Heparin, suggesting that intracellular stored

$345 \mathrm{Ca}^{2+}$ release via $\mathrm{IP}_{3} \mathrm{R}$ played major roles in the TE-induced $\left[\mathrm{Ca}^{2+}\right]_{\mathrm{i}}$ elevation. On the other hand,

346 it was also reported that the $\mathrm{IP}_{3} \mathrm{R}$ could be phosphorylated by various protein kinases, such as

347 PKA and PKC, thus its function might be modulated by these kinases (Morgado et al. 2012). So

348 we next investigated the dependence of PKA and PKC on the $\left[\mathrm{Ca}^{2+}\right]_{\mathrm{i}}$ elevation. Our results 
349 showed that the TE-induced $\left[\mathrm{Ca}^{2+}\right]_{\mathrm{i}}$ rise was significantly attenuated by PKC 412, but not

350 affected by H89, suggesting that the PKC pathway might act on $\mathrm{IP}_{3} \mathrm{R}$ and then cause $\mathrm{Ca}^{2+}$ release,

351 while the PKA pathway seemed to be ineffective in stimulating the $\operatorname{IP}_{3} R$. After that, we further

352 tested the role of $\mathrm{Ca}^{2+}$ in the activation of Akt/eNOS pathway in response to TE. Firstly, our

353 results showed that TE (in $\mathrm{Ca}^{2+}$-containing HBSS) did induce the phosphorylation of both Akt

354 and eNOS, while the phosphorylation level was dramatically decreased by the removal of

355 extracellular calcium, indicating that extracellular $\mathrm{Ca}^{2+}$ was essential to TE-induced eNOS

356 activation. Since we had confirmed that TE-induced $\mathrm{Ca}^{2+}$ mainly came from intracellular stored

$357 \mathrm{Ca}^{2+}$ rather than extracellular $\mathrm{Ca}^{2+}$, we hypothesized that extracellular calcium might be

358 necessary for Akt phosphorylation, and subsequently activate eNOS. In fact, it has been reported

359 that calcium could phosphorylate three kinases (Akt, Erk and Fak) that are involved in the cell

360 survival signalling in neuroblastoma (Satheesh \& Busselberg 2015). However, to determine

361 whether these effects are direct or not, and to clarify the exact effects of extracellular $\mathrm{Ca}^{2+}$ on

362 Akt, further investigations are required. Secondly, we found that TE-induced Akt/eNOS

363 activation was not affected by Nifedipine, suggesting that $\mathrm{Ca}^{2+}$ influx via L-type calcium

364 channels was not involved in TE-evoked Akt/eNOS activation, which was consistent with the

365 results of calcium fluorescent assay where Nifedipine had no influence on the TE-induced $\left[\mathrm{Ca}^{2+}\right]_{\mathrm{i}}$

366 rise. Finally, the phosphorylation of Akt/eNOS was significantly attenuated by the inhibition of 
368 line with the results in fluorescence assay, indicating that TE-induced Akt/eNOS activation

369 might be associated with the calcium release via $\mathrm{IP}_{3} \mathrm{R}$, while $\mathrm{IP}_{3} \mathrm{R}$ was also modulated by $\mathrm{PKC}$

370 pathways rather than PKA pathways. Taken together, these findings confirmed that calcium

371 pathway was also necessary in the activation of TE-induced Akt/eNOS signaling.

\section{5. Conclusions}

In this study, we demonstrated that TE from $C$. capillata could induce dose- and time-

Ser1177 eNOS phosphorylation and activation mainly through PI3K/Akt-dependent, PKC/IP ${ }_{3}$ R-

sensitive and $\mathrm{Ca}^{2+}$-dependent pathway. Since dysfunction of endothelial NO production is one of the major predictors of cardiovascular events, these findings will contribute to a better understanding of the signaling mechanisms of TE in regulating the endothelial function.

Although TE may require further purification and identification in the near future, the current study opens up the possibilities for the development of jellyfish-derived specific-acting drugs that can be used to treat and/or prevent cardiovascular diseases such as hypertension.

\section{Acknowledgements}


384

385

386

387

388

389

390

391

392

393

394

395

396

397

398

399

400

401

402

403

404

405

406

407

408

409

410

411

412

413

414

careful identification of the jellyfish species and Mr. Fang Wei from the Foreign Languages'

Office of the Second Military Medical University for his careful revision of the English language

of the manuscript.

\section{References}

Ayed Y, Dellai A, Ben Mansour H, Bacha H, and Abid S. 2012. Analgesic and antibutyrylcholinestrasic activities of the venom prepared from the Mediterranean jellyfish Pelagia noctiluca. Ann Clin Microbiol Antimicrob 11:15.

Biswas A, Dhar P, and Ghosh S. 2010. Antihyperlipidemic effect of sesame(Sesamum indicum L.) protein isolate in rats fed a normal and high cholesterol diet. Journal of Food Science 75:274-279.

Bloom DA, Burnett JW, and Alderslade P. 1998. Partial purification of box jellyfish (Chironex fleckeri) nematocyst venom isolated at the beachside. Toxicon 36:1075-1085.

Bruschetta G, Impellizzeri D, Morabito R, Marino A, Ahmad A, Spano N, La Spada G, Cuzzocrea S, and Esposito E. 2014. Pelagia noctiluca (Scyphozoa) crude venom injection elicits oxidative stress and inflammatory response in rats. Mar Drugs 12:21822204.

Chen C-C, Ke W-H, Ceng L-H, Hsieh C-W, and Wung B-S. 2010. Calcium- and phosphatidylinositol 3-kinase/Akt-dependent activation of endothelial nitric oxide synthase by apigenin. Life Sciences 87:743-749.

Feng J, Yu H, Li C, Xing R, Liu S, Wang L, Cai S, and Li P. 2010. Isolation and characterization of lethal proteins in nematocyst venom of the jellyfish Cyanea nozakii Kishinouye. Toxicon 55:118-125.

Kerr PM, Tam R, Ondrusova K, Mittal R, Narang D, Tran CH, Welsh DG, and Plane F. 2012. Endothelial feedback and the myoendothelial projection. Microcirculation 19:416-422.

Kim SK, Choi YR, Park PJ, Choi JH, and Moon SH. 2000. Screening of biofunctional peptides from cod processing wastes. J Korean Soc Agric Chem Biotechnol 43:225-227.

Kuhlen JLJ, and Forcucci J. 2012. Angiotensin-converting enzyme inhibitor-induced unilateral tongue angioedema. The American Journal of the Medical Sciences 344:416-417.

Li J, Li Q, Li J, and Zhou B. 2014. Peptides derived from Rhopilema esculentum hydrolysate exhibit angiotensin converting enzyme (ACE) inhibitory and antioxidant abilities. 
415

416

417

418

419

420

421

422

423

424

425

426

427

428

429

430

431

432

433

434

435

436

437

438

439

440

441

442

443

444

445

446

447

448

449

450

Molecules 19:13587-13602.

Lin L, Shun L, and Li BF. 2011. Angiotensin-I-converting enzyme (ACE)-inhibitory and antihypertensive properties of squid skin gelatin hydrolysates. Food Chem 08.064:225230.

Liu G, Zhou Y, Liu D, Wang Q, Ruan Z, He Q, and Zhang L. 2015. Global transcriptome analysis of the tentacle of the jellyfish Cyanea capillata using deep squencing and expressed sequence tags: insight into the toxin- and degenerative disease-related transcripts. PLoS One 10:e142680.

Liu X, Zhang M, Jia A, Zhang Y, Zhu H, Zhang C, Sun Z, and Liu C. 2013. Purification and characterization of angiotensin I converting enzyme inhibitory peptides from jellyfish Rhopilema esculentum. Food Research International 50:339-343.

Liu X, Zhang M, Zhang C, and Liu C. 2012. Angiotensin converting enzyme (ACE) inhibitory, antihypertensive and antihyperlipidaemic activities of protein hydrolysates from Rhopilema esculentum. Food Chem 134:2134-2140.

Morgado M, Cairrão E, Santos-Silva A, and Verde I. 2012. Cyclic nucleotide-dependent relaxation pathways in vascular smooth muscle. Cellular and Molecular Life Sciences 69:247-266.

Nagai H, Takuwa K, Nakao M, Ito E, Miyake M, Noda M, and Nakajima T. 2000a. Novel proteinaceous toxins from the box jellyfish (sea wasp) Carybdea rastoni. Biochem Biophys Res Commun 275:582-588.

Nagai H, Takuwa K, Nakao M, Sakamoto B, Crow GL, and Nakajima T. 2000b. Isolation and characterization of a novel protein toxin from the Hawaiian box jellyfish (sea wasp) Carybdea alata. Biochem Biophys Res Commun 275:589-594.

Noguchi K, Sakanashi M, Matsuzaki T, Nakasone J, Sakanashi M, Koyama T, Hamadate N, and Sakanashi M. 2005. Cardiovascular effects and lethality of venom from nematocysts of the box-jellyfish Chiropsalmus quadrigatus (Habu-kurage) in anaesthetized rats. Toxicon 45:519-526.

Ono S, Hosokawa M, Miyashita K, and Takahashi K. 2006. Inhibition properties of dipeptides from salmon muscle hydrolysate on angiotensin I-converting enzyme. Int $J$ Food Sci Technol 41:383-386.

Park JB, Kario K, and Wang J. 2015. Systolic hypertension: an increasing clinical challenge in Asia. Hypertens Res 38:227-236.

Sammels E, Parys JB, Missiaen L, De Smedt H, and Bultynck G. 2010. Intracellular $\mathrm{Ca}^{2+}$ storage in health and disease: A dynamic equilibrium. Cell Calcium 47:297-314.

Satheesh NJ, and Busselberg D. 2015. The role of intracellular calcium for the development and treatment of neuroblastoma. Cancers (Basel) 7:823-848. 
451

452

453

454

455

456

457

458

459

460

461

462

463

464

465

466

467

468

469

470

471

472

473

474

475

476

477

478

479

480

481
Srivastava K, Bath PM, and Bayraktutan U. 2012. Current therapeutic strategies to mitigate the eNOS dysfunction in ischaemic stroke. Cell Mol Neurobiol 32:319-336.

Suleria HA, Osborne S, Masci P, and Gobe G. 2015. Marine-based nutraceuticals: An innovative trend in the food and supplement industries. Mar Drugs 13:6336-6351.

Tiruppathi C, Minshall RD, Paria BC, Vogel SM, and Malik AB. 2002. Role of $\mathrm{Ca}^{2+}$ signaling in the regulation of endothelial permeability. Vascular Pharmacology 39:173-185.

Vilahur G, Casani L, Mendieta G, Lamuela-Raventos RM, Estruch R, and Badimon L. 2014. Beer elicits vasculoprotective effects through Akt/eNOS activation. Eur J Clin Invest 44:1177-1188.

Wang B, Zhang B, Wang Q, Zhang Z, Nie F, Liu G, Zheng J, Xiao L, and Zhang L. 2013a. Pharmacological studies of tentacle extract from the jellyfish Cyanea capillata in isolated rat aorta. Mar Drugs 11:3335-3349.

Wang T, Wen XJ, Mei XB, Wang QQ, He Q, Zheng JM, Zhao J, Xiao L, and Zhang LM. 2013 b. Lipid peroxidation is another potential mechanism besides pore-formation underlying hemolysis of tentacle extract from the jellyfish Cyanea capillata. Mar Drugs 11:67-80.

Xiao L, He Q, Guo Y, Zhang J, Nie F, Li Y, Ye X, and Zhang L. 2009. Cyanea capillata tentacle-only extract as a potential alternative of nematocyst venom: its cardiovascular toxicity and tolerance to isolation and purification procedures. Toxicon 53:146-152.

Yoshitomi H, Xu Q, Gao M, and Yamori Y. 2011. Phosphorylated endothelial NOS Ser1177 via the $\mathrm{PI} 3 \mathrm{~K} / \mathrm{Akt}$ pathway is depressed in the brain of stroke-prone spontaneously hypertensive rat. Journal of Stroke and Cerebrovascular Diseases 20:406-412.

Zhang CH, Cao WH, Hong PZ, Ji HW, Qin XM, and He JF. 2009. Angiotensin converting enzyme inhibitory activity of Acetes chinensis peptic hydrolysate and its antihypertensive effect in spontaneously hypertensive rats. Int J Food Sci Technol 44:2042-2048.

Zhao Y, Li B, Dong S, Liu Z, Zhao X, Wang J, and Zeng M. 2009. A novel ACE inhibitory peptide isolated from Acaudina molpadioidea hydrolysate. Peptides 30:1028-1033.

Zhuang Y, Sun L, Zhang Y, and Liu G. 2012. Antihypertensive effect of long-term oral administration of jellyfish (Rhopilema esculentum) collagen peptides on renovascular hypertension. Eur J Clin Invest 10:417-426. 